\title{
Evaluating the Impact of Transmission Range on the Performance of VANET
}

\author{
Akram A. Almohammedi ${ }^{1}$, Nor K. Noordin ${ }^{2}$, Sabri Saeed ${ }^{3}$ \\ ${ }^{1,2}$ Department of Computer and Communication Systems Engineering, Universiti Putra Malaysia, Malaysia \\ ${ }^{3}$ Department of Electrical, Electronic and Systems Engineering, Universiti Kebangsaan Malaysia, Malaysia
}

\begin{abstract}
Article Info
Article history:

Received Sep 22, 2015

Revised Dec 8, 2015

Accepted Jan 2, 2016

\section{Keyword:}

End to end delay

Packet delivery ratio

Transmission range

VANET

ABSTRACT

Recently, interest in the field of Vehicular Ad-hoc Networks (VANETs) has grown among research community to improve traffic safety and efficiency on the roads. Despite the many advantages, the transmission range in vehicular network remains one of the major challenges due to the unique characteristics of VANETs such as various communication environments, highly dynamic topology, high node mobility and traffic density. The network would suffer from a broadcast-storm in high vehicular density when a fixed transmission range in VANET is used, while in sparse vehicular density the network could be disconnected frequently. In this paper, we evaluated the impact of different transmission ranges and number of flows formed between vehicles in a highway scenario using AODV as routing protocol. In order to validate the simulation of VANET, traffic and network simulators (SUMO \& NS-2) have been used. The performance was evaluated in terms of packet delivery ratio and end-to-end delay. The simulation results have shown that better performance was achieved in term of higher PDR and lower end-to-end delay for less than 500 meters transmission range. On the contrary, the PDR started to decrease and end-to-end delay increased when the transmission range exceeded 500 meters. The performance degraded as the number of flows increased.
\end{abstract}

Copyright (C) 2016 Institute of Advanced Engineering and Science. All rights reserved.

\section{Corresponding Author:}

Akram A. Almohammedi,

Department of Computer and Communication Systems Engineering,

Universiti Putra Malaysia,

43300 Serdang, Malaysia.

Email: Akram.a.a@ieee.org

\section{INTRODUCTION}

Recently, Vehicular Ad-hoc Networks (VANETs) have attracted great attention among governmental organizations, industrial sectors, and academic institutions due to their significant applications. It can be utilized mainly to improve vehicle safety, enhance traffic management conditions and provide infotainment in vehicles such as Internet access, video streaming, etc. However, VANETs are a sub-class of Mobile Ad-hoc Networks (MANETs) with several different characteristics that distinguish them from other MANETs, such as large number of nodes, high mobility, change rapidly on network topology, no power constraints and availability of GPS [10]. Thereby, the medium access control (MAC) protocols presented for MANETs are not fit for VANETs, owing to the unique features of VANETs. In designing the MAC protocols for VANETs, one should consider a specific way for the nodes to share the underlying channel, as well as the type of message [1].

VANETs are considered as a type of distributed self-organizing network among vehicles equipped with wireless communication devices to provide communication among nearby vehicles. This type of communication is known as intelligent transportation System (ITS) application. The communication can either be between vehicles (V2V), or between vehicles and the roadside infrastructure (V2I) to generate 
significant improvement to the transportation system performance. The significant objective of communication between vehicles is to disseminate alert messages to neighbor vehicles about an accident, a bad weather or to communicate with RSU to obtain the traffic light status. However, VANET acquires sensors to be embedded on the vehicles and infrastructures [1]. Moreover, communication between vehicles can be developed by integration of embedded computers supported with sensing devices, digital maps, navigation systems (GPS) and wireless communication devices as well. Each vehicle has On Board Unit (OBU) to provide communication with another OBU that merges to another vehicle or with Road Side Units (RSU) that is installed at a road side [19].

The proposed technology to perform this kind of communication is Dedicated Short Range Communication (DSRC). DSRC is a wireless technology developed based on Wi-Fi to support the information exchange between V2V and V2I, because it is designed for very high dynamic networks. These communication networks have $75 \mathrm{MHz}$ frequency spectrum in the range of5.9 $\mathrm{GHz}$ allocated by the Federal Communications Commission (FCC) [5]. The $75 \mathrm{MHz}$ frequency spectrum of the DSRC is divided into seven channels, $10 \mathrm{MHz}$ for each channel and $5 \mathrm{MHz}$ for guard band. One of the channels is the control channel (CCH_178) and the other six are service channels (SCHs) [11]. In particular, the control channel is used for safety applications while the other six channels are used for non-safety applications. Furthermore, another standard merged within this protocol is Wireless Access for Vehicular Environment (WAVE) to work in the upper layer. WAVE is a new standard developed based on the IEEE 802.11p and the IEEE 1609 standards. The 802.11p or WAVE is a major area of interest in the field of research and development to provide enhancements on the physical (PHY) and medium access control (MAC) layers of the 802.11 protocol. IEEE 1609 family has sub-detailed standards that include IEEE 1609.1, IEEE 1609.2, IEEE 1609.3, and IEEE 1609.4, used for remote management services, security services, network services and multichannel operations, respectively [11].

However, a typical transmission range in VANETs has not been specified yet, although its standard proposed a range for distance up to $1 \mathrm{~km}$. Subsequently, the transmission range is considered as one of the obstacles in vehicular networks. The reason lies in the unique features of VANETs in terms of, various communication environments, highly dynamic topology, high node mobility, propagation model and potentially large scale. Therefore, VANETs are prone to suffer from a broadcast-storm in high vehicular density as a result of using a fixed transmission range especially in urban areas, while in sparse vehicular density (e.g., highway) the network could be disconnected frequently.

As derived from the literature study, a considerable amount of researchers have discussed this issue, of those include [2], [3], [7], [8], [12], [14], [15], [22]. In [2], the authors discussed the comparison in the performance of Ad-hoc On-Demand Distance Vector (AODV) routing protocol with Optimized Link State Routing protocol (OLSR) on two different road network scenarios. The first road scenario is a complex road network which represents the city road network with multiple crossroads, and the other is an intersection of two roads. The main objective of the study was to do an assessment on the applicability of AODV and OLSR protocols in VANET with different traffic scenarios and transmission ranges of IEEE 802.11p standard. The V2V connectivity for vehicular communication in fading channels was introduced by [3]. The vehicles were considered traveling in opposite or similar direction. From the results, it was known that the connectivity in VANET can be improved by adapting the transmission range based on the predicted local vehicle density (and velocity), as well as switching into a less-congested frequency band. The connectivity of a Vehicular Ad hoc Network (VANET) formed between vehicles that move on a highway was scrutinized in [12]. The authors also discussed the analytical model to determine the network connectivity of vehicular network by assuming vehicle speeds follow normal distribution. The results illustrate increment of network connectivity when the transmission range of vehicles increased. On the contrary, the network connectivity degraded with increasing average vehicle speed. It is known from the study that increasing the average speed would increase the critical transmission range required to meet a given connectivity probability criterion. The effect of wireless transmission range on the lifetime improvement of the routing path in VANET was presented by [14]. The model has taken unicast routing and number of vehicles moving on the lane having their respective speed. The results showed that increasing the wireless transmission range can improve the reliability of the routing path in an Inter-vehicular communication network. Also, the path setup probability in an Intervehicular communication network can be increased as the wireless transmission range is increased.

The work of [15] proposed a novel broadcast algorithm known as Transmission Range Adaptive Broadcast (TRAB) derived from the location-based ideas for vehicles with different transmission ranges. The simulation results showed that even in the case of different transmission ranges from different vehicles, TRAB can significantly bring lower dissemination redundancy as to improve broadcast efficiency, and guarantees better real-time performance and reliability of message dissemination. In [22], the authors specifically focused on the transmission power and its effect on UDP throughput in vehicular networks. The authors found that throughput was mainly influenced by the number of hops between the source and the 
destination. Consequently, increasing the transmission range decreased the number of hops between source and destination. Theoretically, as the transmission range is correlated to the average number of hops, lesser hops would effectively increase throughput. The study concluded, increasing the transmission range until a certain point (i.e. 1000 meter) saturates the throughput due to higher interference. They realized that the effect of vehicle density is only important at lower transmission ranges to provide the required connectivity.

In this paper we studied the interaction of AODV for VANETs under different transmission ranges with varying data rates and number of flows. This study evaluated particular vehicles with certain mobility and broadcasting messages synchronously using the wireless access environments available in our scenario. At the end of simulation, the packet delivery ratio and end-to-end delay were calculated. Rest of the paper is organized as follows: The DSRC AND WAVE STANDARDS are presented in Section 2. In section 3, we demonstrate the simulations used in this study. Section 4 contains the results and analysis. The paper is concluded in Section 5.

\section{DSRC AND WAVE STANDARDS}

Dedicated Short Range Communication (DSRC) is a wireless technology developed based on Wi-Fi to support information exchange amongV2V and V2I [11]. DSRC has been designed to be exploited in automotive industry. It is a set of protocols and standards containing all parts of layers, including the PHY layer and application layer for VANET [18]. American Society for Testing and Materials (ASTM) subcommittee (E17.51) is the organization that has started to work on the DSRC standard. Thus, ASTM has the authority to manage the issues in vehicle roadside communications [9]. The standard version, E2213-03 (ASTM 2003) for DSRC was published in July 2003 by ASTM. This standard relies on the IEEE 802.11a protocol by merging with some editing on PHY and MAC layers specified in IEEE 1999 and 2003, respectively. DSRC is a licensed spectrum and can be used without any charges as permitted by the Federal Communications commission (FCC) [9].

Despite the free usage band, a few restricted rules which were issued by the FCC in 2004are emphasized. Moreover, DSRC is used in a highly dynamic network to provide reliable communication with minimum latency. Thereby, DSRC is dedicated for short time responses. The communication network for DSRC has $75 \mathrm{MHz}$ of the frequency spectrum in the range of $5.9 \mathrm{GHz}$ allocated by the FCC for distance up to $1 \mathrm{~km}$ to support safety and non-safety applications [5]. As mentioned before, the $75 \mathrm{MHz}$ frequency spectrum of the DSRC is divided into seven channels with $10 \mathrm{MHz}$ each, and $5 \mathrm{MHz}$ for guard band as shown in figure 1 . One of these channels is called control channel (CCH_178) and the other six are service channels (SCHs) [11]. Moreover, the control channel is used for safety applications while the others are for non-safety applications. Safety applications are given higher priority over non-safety applications.

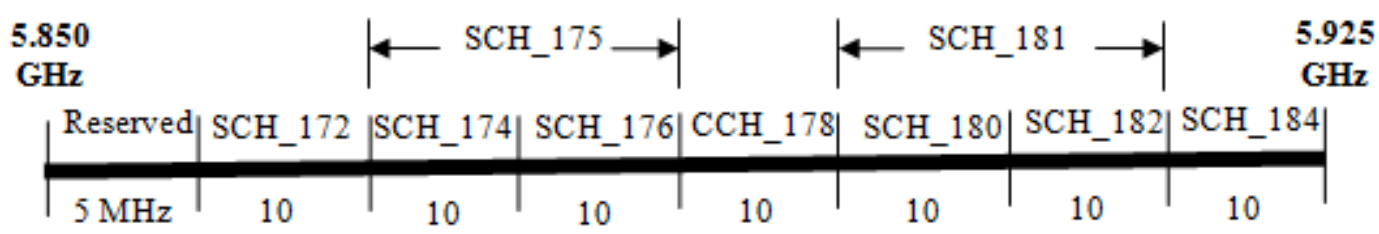

Figure 1. DSRC spectrum band and channels in the U.S.

The DSRC supports different types of transfer rate such as 3, 4.5, 6, 9, 12, 18, 24 and 27 Mbps for a $10 \mathrm{MHz}$ channel. The most optimal transfer rate in DSRC is $6 \mathrm{Mbps}$ as in [20]. Two specific channel pairs are able to be merged with a $20 \mathrm{MHz}$ channel to support transfer rates of $54 \mathrm{Mbps}$ in special conditions: channel 174 with 176, and channel 180 with182. In addition, Wireless Access in Vehicular Environment (WAVE) is a new standard, designed for VANET to support a CCH and multiple SCHs. However, WAVE interface was developed based on the IEEE 802.11p and IEEE 1609 standards to operate on Physical (PHY) layer, Medium Access Control (MAC) layer and higher layer protocols [11], where IEEE 802.11p is the base layer standard and IEEE 1609 is the upper layer standard [19]. Meanwhile, IEEE 1609 family is categorized into four standards as follows: IEEE 1609.1[23], IEEE 1609.2 [6], IEEE 1609.3 [16], and IEEE 1609.4 [13], often used for remote management services, security services, network services and multi-channel operations respectively as in figure 2. The channel access time in WAVE stack was represented in figure 3, which is evenly divided into repeating synchronization (sync) intervals of $100 \mathrm{~ms}$ [13], and each sync interval is divided into $\mathrm{CCH}$ Intervals (CCHI) of $50 \mathrm{~ms}$ and $\mathrm{SCH}$ Intervals (SCHI) of $50 \mathrm{~ms}$. In addition, the standard 
defines a Guard interval at the beginning of each channel interval. Typical values for the guard interval are between 4 and $6 \mathrm{~ms}$, using for radio switching and not for transmissions. Synchronization between vehicles is achieved by receiving the coordinated universal time (UTC) provided by the navigation satellite systems (GPS) equipped in each vehicle.

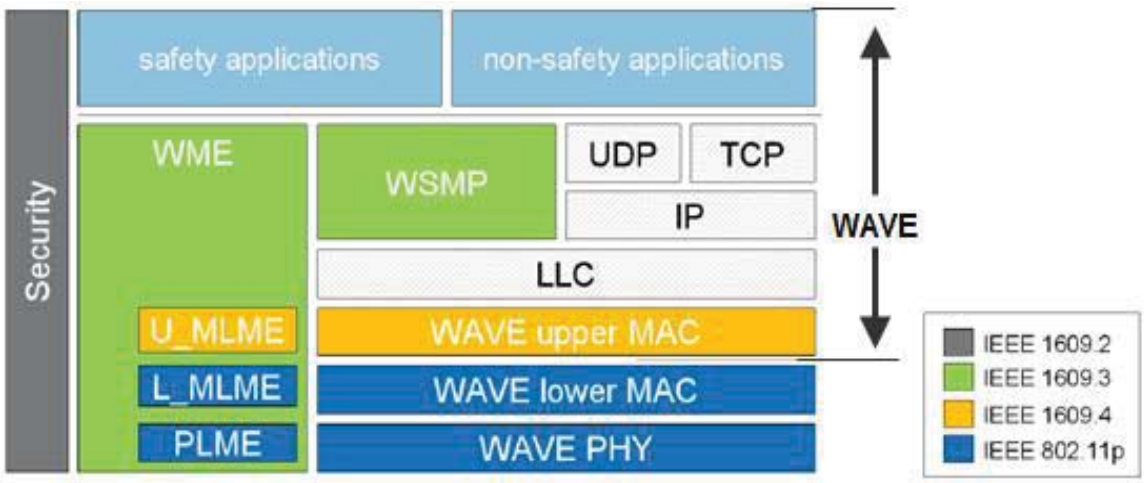

Figure 2. DSRC and WAVE standards architecture

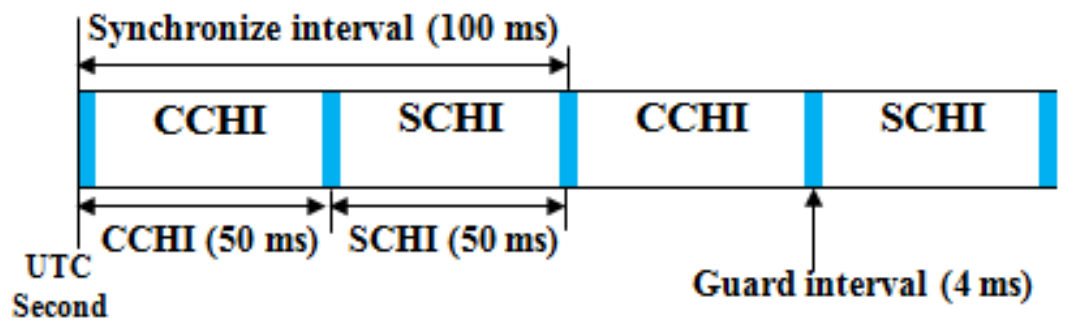

Figure 3. Sync interval, guard interval, $\mathrm{CCH}$ interval, and SCH interval

The IEEE 802.11p standard defines the PHY and MAC layers of the WAVE architecture protocol. It is based on the 802.11 standard which was established in 2010 [5]. Furthermore, since IEEE 802.11p is the amendment of IEEE 802.11 Distributed Coordination Function (DCF), thus, 802.11p uses the Enhanced Distributed Channel Access (EDCA) as the MAC method. EDCA uses the Carrier Sense Multiple Access/Collision Avoidance (CSMA/CA) method for accessing the shared medium. The operation method of CSMA/CA is by listening to the channel at first. Therefore, if the channel is free for an arbitration inter-frame space (AIFS), the node starts transmitting directly, if it is busy or becomes busy during the AIFS, the node must perform a back-off [21]. The main purpose of the IEEE 802.11p standard is to give channel access through the EDCA by supporting different types of Access Categories (ACs) which has a range from AC0 to AC3, from the lowest to the highest priority as in table 1 [17]. At the MAC sub-layer, the IEEE 802.11p standard provides a different role. It defines an extremely low overhead WAVE Basic Service Set (WBSS) to form groups of vehicles communicating at the same area to support V2V and V2I communications for safety and non-safety applications [4].

Table 1. Default EDCA parameters in IEEE P802.11p [17].

\begin{tabular}{llccc}
\hline AC No. & Access Class & CW $_{\min }$ & CW $_{\max }$ & AIFSN[AC] \\
\hline 0 & Background traffic (BK) & 15 & 1023 & 9 \\
1 & Best Effort (BE) & 7 & 15 & 6 \\
2 & Voice (VO) & 3 & 7 & 3 \\
3 & Video (VI) & 3 & 7 & 2 \\
\hline
\end{tabular}




\section{SIMULATIONS}

To have a feasible scenario for the system, a computer-based simulation is necessary. Our simulation program aimed to study the impact of transmission range for VANET protocol and to evaluate its performance under different transmission ranges with varying data rates and number of flows. For this purpose, a traffic scenario of a highway was designed using Simulation of Urban Mobility (SUMO) [24]. NS2 [25] was utilized as the network simulator to validate the results. Simulations were conducted for 802.11p based on CSMA/CA algorithm for the CSMA/CA of algorithm. Moreover, the length of highway was $8 \mathrm{~km}$ with 6 lanes in both directions which comprises 3 lanes in each direction. Particularly, the scenario of a highway was built because the environment was suitable for investigating the biggest challenges of the MAC layer. One hundred nodes (vehicles) with speed $60 \mathrm{~km} / \mathrm{h}$ were tested in the scenario. The simulated sensing ranges were $100-700 \mathrm{~m}$. Four different data rates were being considered including 64,100,250 and 512 Kbyte. AODV was the selected routing protocol for the simulation and the behavior of AODV protocol was evaluated in terms of packet delivery ratio and end-to-end delay. The most optimal transfer rate to be supported by 802.11pwas $6 \mathrm{Mbps}$ as according to [20].Table 2 shows the parameters of simulation settings. In order to describe briefly the execution of the program, initially we selected the network type (i.e. VANET) to build the scenario (i.e. highway), followed by setting the initial values to be used for the model. The initial values should be inserted first into the traffic simulator (i.e. SOMU) to create nodes mobility for the proposed model. The movement details were generated using SOMU/MOVE. Furthermore, the mobility file that has been achieved by the traffic simulator was converted and sent to the network simulator (NS-2) to configure the network by assigning UDP, AODV, and IEEE 802.11p MAC protocol. In this case the nodes connectivity should be initiated. With respect to the nth simulation cycles for the model, the transmission range, nodes connectivity and traffic load were changed constantly to study the impact of different transmission ranges and the number of flows in the network.

In fact, the single line-of-sight path between two mobile nodes was rarely in a wireless communication; consequently the two-ray ground reflection model which deals with both the direct path and the ground reflection path has been considered in our work. The received power at a certain distance was calculated as follows:

$$
\operatorname{Pr}=\frac{P t * G t * G r * h t^{2} * h r^{2}}{d^{4} * L}
$$

given that: Gt and Gr are the gain of the transmitter and receiver antennas, respectively; ht and hr are the heights of the transmitter and receiver antennas; $d$ is the distance between the receiver and the transmitter; and $\mathrm{L}$ is the system loss, often around 1.

There are two common types of evaluation criteria studied, which are Packet delivery ratio and end-to-end delay.

$$
D=\left(D_{M A C}+D_{P}\right) * N
$$

where $\mathrm{D}, D_{M A C}, D_{P}$ and $\mathrm{N}$ are delay, MAC delay, propagation delay and hop counts, respectively.

Table 2. Simulation parameters

\begin{tabular}{ll}
\hline Parameters & Specification \\
\hline MAC Protocol & IEEE 802.11P \\
Propagation model & TwoRayGround \\
Routing protocol & AODV \\
Packet type & CBR \\
Transmission protocol & UDP \\
Highway length & $8 \mathrm{~km}$ \\
No. of lanes & $6(3$ in each direction $)$ \\
Width of each lane & $4 \mathrm{~m}$ each \\
No. of nodes & 100 \\
Node speed & $60 \mathrm{~km} / \mathrm{h}$ \\
Packet length & $64,100,250 \& 512 \mathrm{~Kb}$ \\
Packet size & 1000 \\
Bandwidth & $6 \mathrm{Mbps}$ \\
Transmission range & $100-700$ meter \\
Time of simulation end & $1796 \mathrm{~s}$ \\
&
\end{tabular}




\section{RESULTS AND ANALYSIS}

This section emphasized and discussed the simulation results. As the goal of this paper was to scrutinize the behavior of VANET protocol with respect to different transmission ranges, traffic loads and number of flows, the evaluation was focused on two common performance metrics, i.e. packet delivery ratio and end-to-end delay. The results were described briefly in the following sections, along with figures that exhibit the analyzed results in terms of the performance metrics.

\subsection{Packet Delivery Ratio (PDR)}

The following figures depict the behavior of VANET protocol based on PDR. Figure 4 shows the performance of VANET protocol using AODV as routing protocol with respect to the different transmission ranges: 100, 200, 300, 400, 500, 600 and 700 meters. The results imply that PDR is in direct variation with up to $500 \mathrm{~m}$ transmission range which suggests increasing PDR in correlation to higher transmission range. This increment has affected the reduction in the number of hops and sufficient connectivity by covering a wide area with higher power signal to ensure that nearly every packet arrives at the destination successfully. In contrast, when the transmission range was over $500 \mathrm{~m}$ e.g. $600 \mathrm{~m}$ and $700 \mathrm{~m}$, the PDR decreased that was probably due to the higher contention flows which had caused higher interference rate. Nonetheless, the rules of CSMA/CA restrict many nodes from communication by using carrier sense that limits the reuse of the bandwidth. Relatively, when the transmission range was $100 \mathrm{~m}$ the value of PDR was low i.e. 0.31 that reflects upon the influence of number of hops between the source and destination towards the PDR. Consequently, decreasing the transmission range would increase the number of hops and led to disconnectivity between the source and destination.

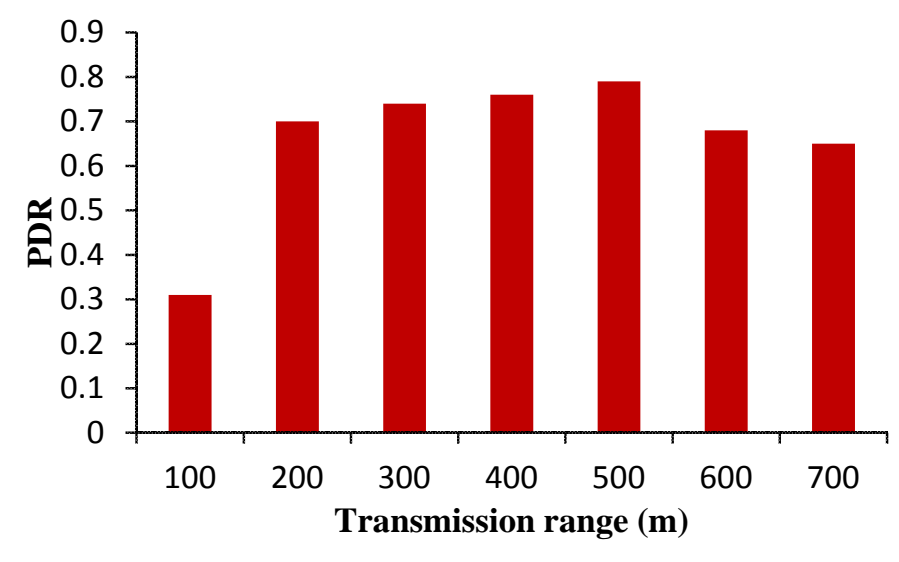

Figure 4. Packet delivery ratio versus transmission range (meter)

The remaining figures were obtained from the tests using the average and highest protocol performance from figure 4 i.e. 300and 500 meters. These figures focus on describing the effect of number of flow variations and data rates under $300 \mathrm{~m}$ and $500 \mathrm{~m}$ transmission ranges. Figures 7(a) \& 6(b) describe the PDR for certain flows using AODV as routing protocol. A notable observation is the decreasing protocol performance around several flows for $300 \mathrm{~m}$ transmission range. Conversely, when the transmission range was increased to $500 \mathrm{~m}$, the performance of the protocol increased in terms of the PDR. This indicates that when the transmission range was $300 \mathrm{~m}$, the packet dropped and disconnectivity increased, owing to the increasing hops between the source and destination to deliver the data, as well as sparse vehicular density. Figure 5(a) shows that the PDR is 0.8 for flow 1 and 0.2 for flow 5 in conjunction to the transmission range of $300 \mathrm{~m}$; this was due to the sparse vehicular density which caused disconnectivity in flow 5 . 


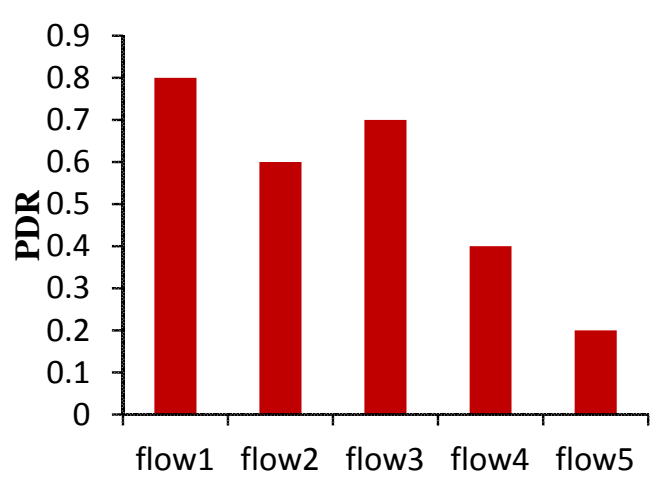

(a)

flow

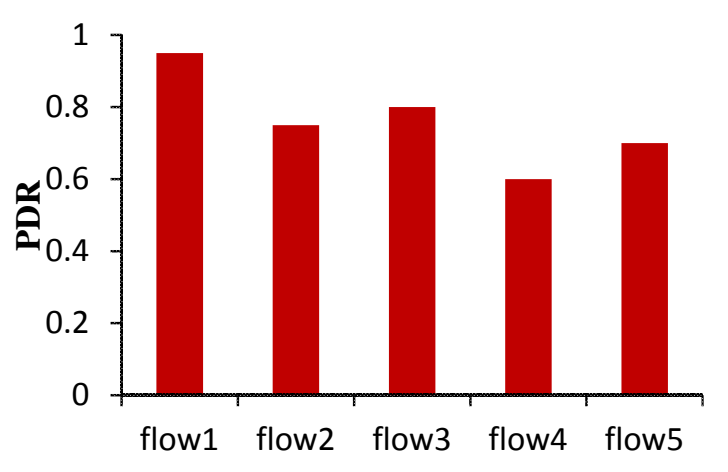

(b)

flow

Figure 5. (a) Packet delivery ratio to each flow at $\operatorname{Tr}=300 \mathrm{~m}$. (b) Packet delivery ratio to each flow at $\mathrm{Tr}=$ $500 \mathrm{~m}$

Similarly, figure 6 shows the PDR from different number of flows under transmission range of 300 $\mathrm{m}$ and $500 \mathrm{~m}$. As noticed from the figure, the transmission range has a positive correlation with performance of the protocol. Generally, higher number of flows reduces the PDR due to the exponential proliferation upon the number of collisions as a result of higher contention flows. As can be observed from the figure below, under transmission range of $500 \mathrm{~m}$, PDR decreases when the number of flows increases. For instance, when the number of flows is2, the PDR is 0.8 and decreased to 0.6 when the number of flows is 20 . One unanticipated finding was observed, of which initially the PDR was 0.77 with 5 flows and it increased slightly to 0.8 during 10 flows. A reasonable explanation could be because with the 10 flows, the vehicles might not be in each other's transmission range and the vehicular density was high to maintain the connectivity. Somehow, with 5 flows the vehicles were in the transmission range of each other which led to higher interferences.

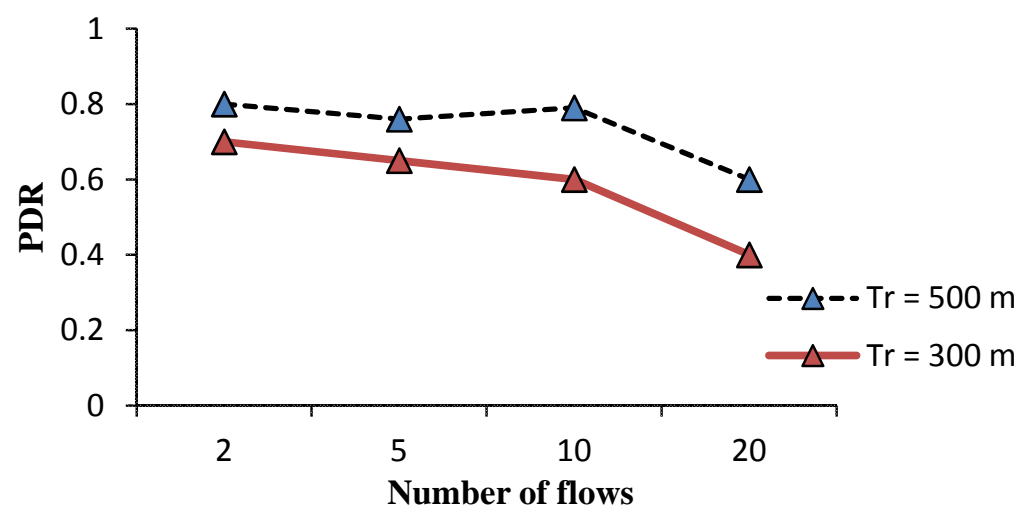

Figure 6. Packet delivery ratios vs. number of flows

\subsection{End-to-End Delay}

In order to evaluate the behavior of VANET protocol in term of end-to-end delay, the following figures were plotted. Figure 7 illustrates the performance of VANET protocol in term of delay by using AOVD as routing protocol with respect to the different transmission ranges i.e. 100, 200, 300, 400, 500, 600, and $700 \mathrm{~m}$. Basically, as the number of hops increases the delay increases as well. Therefore, when the transmission range was high, the number of hops and delay decreased. Figure 7 displays decreasing delay as the transmission range was increased up to $500 \mathrm{~m}$. This is due to the decreasing hops along with a high vehicular density which led to sufficient connectivity between sources and destinations. On the contrary, when the transmission range was $100 \mathrm{~m}$ the delay was very high due to the increasing hops (MAC and propagation delays) for data to be reached at a destination. In addition, when the transmission range reached 
certain point i.e. above $500 \mathrm{~m}$, for instance $600 \mathrm{~m}$, the delay began to increase slowly which was associated to the MAC delay from contended data sending from nodes, producing higher collisions and interferences.

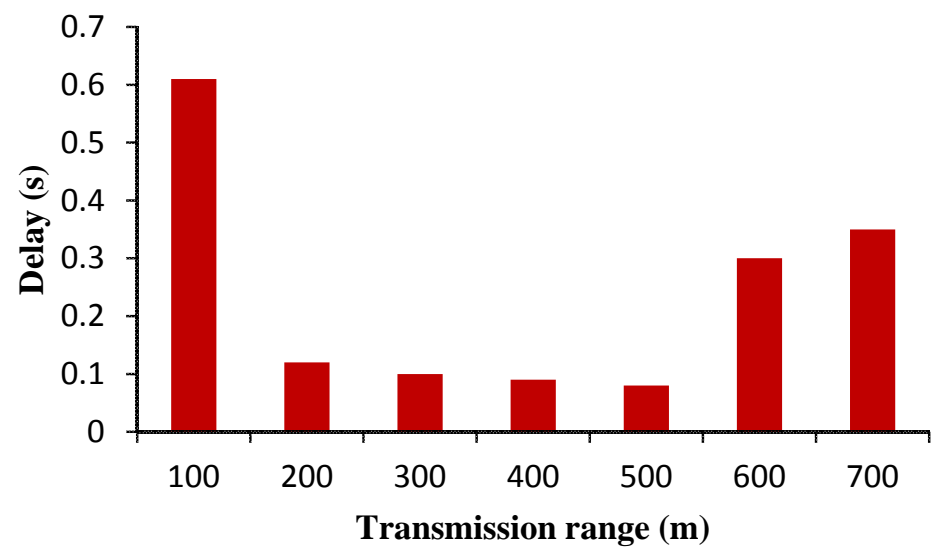

Figure 7. Delay versus transmission range (meter)

Figure 8(a) represents the delay of VANET protocol for transmission range of $300 \mathrm{~m}$. Nonetheless, the delay was high with most of the flows, owing to the increased hops between source and destination. The high delay was also the effect from sparse vehicular density which led to frequent disconnectivity. The behavior of VANET protocol in term of delay for $500 \mathrm{~m}$ transmission range is presented in Figure 8(b). In particular, we noticed very low delays in most cases such as in flow2, flow 4 and flow 5 with delays $0.05 \mathrm{~s}$, $0.01 \mathrm{~s}$ and $0.02 \mathrm{~s}$, respectively. This was possible as higher transmission ranges covered wider area, thus decreased the number of hops which enabled constant and sufficient connectivity among vehicles. As it can be observed in Figure 8(b), there is an unusual display for flow1, of which the delay is very high (0.55 s). This outcome was due to the large distance between the sender and receiver that had increased the number of hops. Overall, Figures 10(a) \&10(b) have proven the inverse relationship of transmission range and the delay.
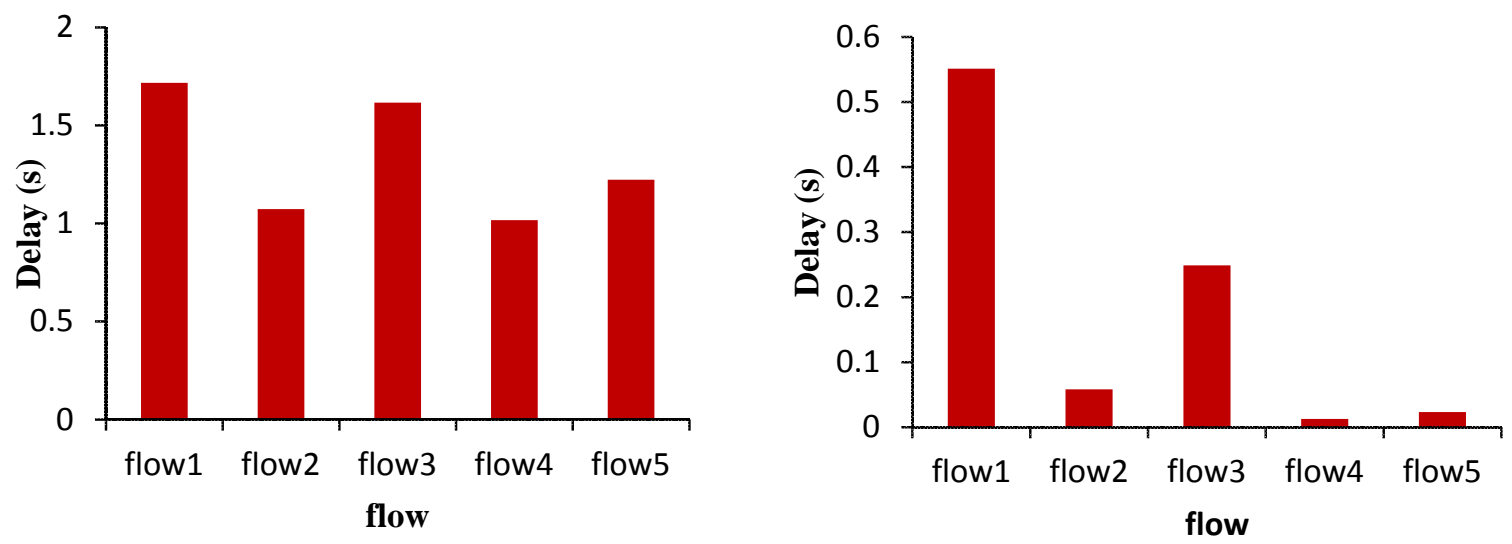

Figure 8. (a) Delay in each flow at $\operatorname{Tr}=300 \mathrm{~m}$. (b) Delay in each flow at $\operatorname{Tr}=500 \mathrm{~m}$

Figure 9 below shows the performance of the protocol in term of average end-to-end delay with respect to the different transmission ranges and number of flows. Nevertheless, as clearly seen from Figure 9 the transmission range increased when the average end-to-end delay decreased, and vice versa. On the other hand, when the number of flows was increased, the average end-to-end delay also increased. This is due to the higher contention flows among the nodes that led to higher interferences. Taking the $500 \mathrm{~m}$ transmission range as an example, when the number of flows was 2, the average end-to-end delay was $0.3 \mathrm{~s}$, and when it was 20 , the average end-to-end delay was $1.8 \mathrm{~s}$. This was in accordance to the high frequency of collisions and interferences as the number of flows increased. 


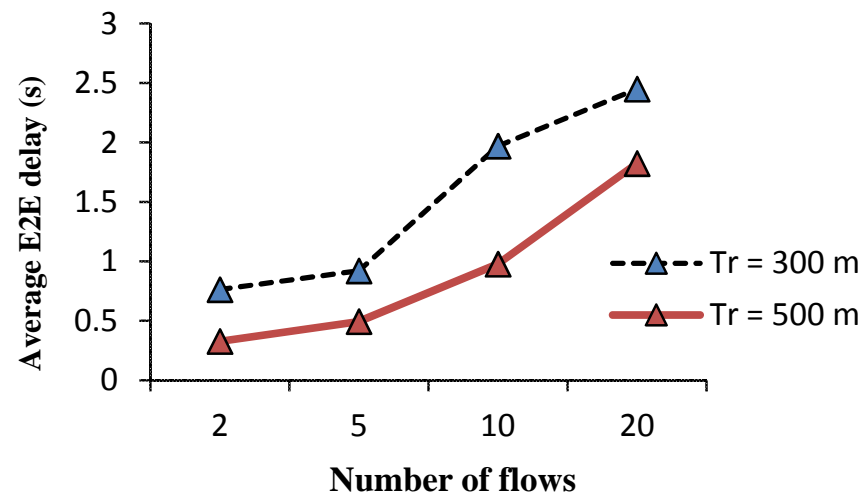

Figure 9. Average end to end delays versus number of flows

\section{CONCLUSION}

The incorporation of computing, telecommunications (fixed and mobile), and various kinds of services are facilitating the deployment of different types of VANET technologies. Recently, vehicular network projects have been taken into consideration by many researchers around the world, and several VANET standards have been developed to improve V2V or V2I communications. Hence, to understand the behavior of VANETs, this paper had generated a highway scenario. The main goal of this paper was to evaluate the impact of different transmission ranges and number of flows that change rapidly in VANET environment using AODV as routing protocol. In order to validate the simulation of VANET, traffic and network simulators (SUMO \& NS-2) were used. The performance was evaluated in terms of packet delivery ratio and end-to-end delay. The simulation results show that better performance can be achieved in term of higher PDR and lower end-to-end delay by lowering the transmission range of less than 500 meters. On the contrary, when the transmission range was more than 500 meters, PDR will start to decrease and end-to-end delay will increase. The performance degraded as the number of flows increased.

\section{REFERENCES}

[1] Gillani SA, Shah PA, Qayyum A, Hasbullah HB. MAC Layer Challenges and Proposed Protocols for Vehicular Ad-hoc Networks. Vehicular Ad-hoc Networks for Smart Cities: Springer; 2015. p. 3-13.

[2] Jaiswal RK, Jaidhar C. An Applicability of AODV and OLSR Protocols on IEEE 802.11 p for City Road in VANET. Internet of Things, Smart Spaces, and Next Generation Networks and Systems: Springer; 2015. p. 28698.

[3] Rawat DB, Shetty S, editors. Enhancing connectivity for spectrum-agile vehicular ad hoc networks in fading channels. Intelligent Vehicles Symposium Proceedings, 2014 IEEE; 2014: IEEE.

[4] Torabi N, Ghahfarokhi BS, editors. Implementation of the IEEE $802.11 \mathrm{p} / 1609.4$ DSRC/WAVE in NS-2. Computer and Knowledge Engineering (ICCKE), 2014 4th International eConference on; 2014: IEEE.

[5] Palma V, Vegni AM. On the Optimal Design of a Broadcast Data Dissemination System over VANET Providing V2V and V2I Communications" The Vision of Rome as a Smart City". Journal of Telecommunications and Information Technology. 2013:41-8.

[6] IEEE Standard for Wireless Access in Vehicular Environments Security Services for Applications and Management Messages, IEEE Std 1609.2-2013, April 2013.

[7] Godbole V. Intelligent driver mobility model and traffic pattern generation based optimization of reactive protocols for vehicular ad-hoc networks. International Journal of Information and Network Security (IJINS). 2013;2(3):20715.

[8] Ying W, Hui-bin X, Dai-feng C. A novel routing protocol for VANETS. TELKOMNIKA Indonesian Journal of Electrical Engineering. 2013;11(4):2195-9.

[9] Zeadally S, Hunt R, Chen YS, Irwin A, Hassan A. Vehicular ad hoc networks (VANETS): status, results, and challenges. Telecommunication Systems. 2012;50(4):217-41.

[10] Piran MJ, Murthy GR, Babu GP. Vehicular ad hoc and sensor networks; principles and challenges. arXiv preprint arXiv:11082776. 2011.

[11] Rawashdeh ZY, Mahmud SM. Communications in Vehicular Networks. Wayne State University. Detroit; 2011.

[12] Muhammed A, Neelakantan P, Babu A, editors. Network Connectivity of One-dimensional Vehicular Ad Hoc Network. Proceedings of International Conference on Communications and Signal Processing Kerala, India: IEEE Press; 2011.

[13] IEEE Standard for Wireless Access in Vehicular Environments (WAVE) - Multi Channel Operation, IEEE Std 1609.4-2010, February 2011. 
[14] Dorle SS, Khandare S, Keskar AG, Chakole MB, editors. Wireless Transmission Impact on the Lifetime of Routing Path in VANET. Emerging Trends in Engineering and Technology (ICETET), 2010 3rd International Conference on; 2010: IEEE.

[15] Xue-Wen W, Wei Y, Shi-Ming S, Hui-bin W, editors. A transmission range adaptive broadcast algorithm for vehicular ad hoc networks. Networks Security Wireless Communications and Trusted Computing (NSWCTC), 2010 Second International Conference on; 2010: IEEE.

[16] IEEE Standard for Wireless Access in Vehicular Environments (WAVE) - Networking Services, IEEE Std 1609.32010, December 2010.

[17] Association IS. 802.11 p-2010 - ieee standard for information technology —local and metropolitan area networksspecific requirements-part 11: Wireless lan medium access control (MAC) and physical layer (PHY) specifications amendment 6: Wireless access in vehicular environments. URL http://standards ieee org/findstds/standard/80211 p-2010 html.

[18] Hartenstein H, Laberteaux K. VANET vehicular applications and inter-networking technologies: John Wiley \& Sons; 2009.

[19] Moustafa H, Senouci SM, Jerbi M. Introduction to Vehicular Networks. 10th November. 2008.

[20] Jiang D, Chen Q, Delgrossi L, editors. Optimal data rate selection for vehicle safety communications. Proceedings of the fifth ACM international workshop on VehiculAr Inter-NETworking; 2008: ACM.

[21] Bilstrup K, Uhlemann E, Ström EG, Bilstrup U, editors. Evaluation of the IEEE 802.11 p MAC method for vehicleto-vehicle communication. Vehicular Technology Conference, 2008 VTC 2008-Fall IEEE 68th; 2008: IEEE.

[22] Khorashadi B, Chen A, Ghosal D, Chuah C-N, Zhang M, editors. Impact of transmission power on the performance of UDP in vehicular ad hoc networks. Communications, 2007 ICC'07 IEEE International Conference on; 2007: IEEE.

[23] IEEE Draft Trial-Use Standard for Wireless Access in Vehicular Environments (WAVE) - Resource Manager, IEEE Std 1609.1-206, October 2006

[24] www.isi.edu/nsnam/ns

[25] http://sumo.sourceforge.net/ 